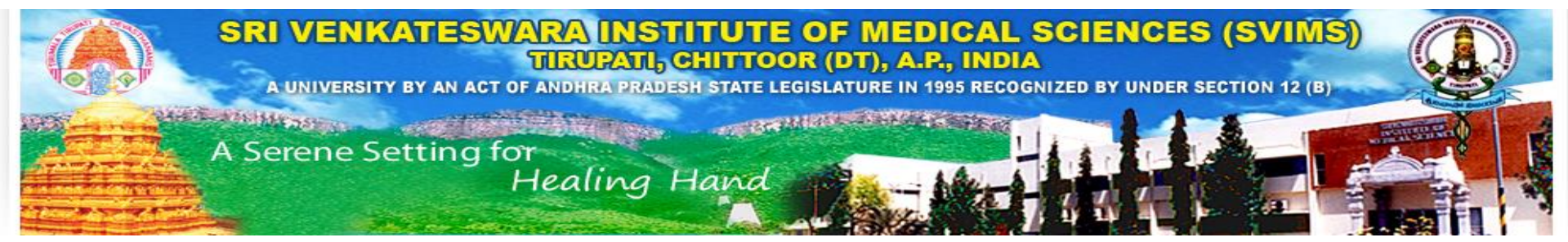

\title{
TITLE: ASSESSMENT OF INTENSITY OF CHOLESTEROL LOWERING BY LOW DOSE ATORVASTATIN IN PATIENTS WITH TYPE 2 DIABETES MELLITUS.
}

\section{AUTHORS:}

Ashok Venkatanarasu ${ }^{1}$, Vaikkakara Suresh ${ }^{1}$, Alok Sachan ${ }^{1}$, Nimmanapalli Harini Devi ${ }^{2}$

1. Dept. of Endocrinology 2. Dept. of Biochemistry.

Sri Venkateswara Institute of Medical Sciences, Tirupati, A.P. India.

\section{Background:}

Cardiovascular disease (CVD) is leading cause of morbidity and mortality in Diabetic patients ${ }^{1}$, which requires integrated approach including management of dyslipidemia. Recent ACC/AHA guidelines recommend either moderate or high intensity of statin therapy ${ }^{2}$ but not particular target levels in these patients.

Aims:

To determine the proportion of patients with type 2 diabetes mellitus (T2DM) achieving AHA/ACC definitions of moderate or high intensity reduction of low density lipoprotein cholesterol (LDL-C) with low dose (10mg/day) of atorvastatin.

\section{Methods:}

It was a retrospective study. All patients with T2DM with lipid profile at baseline and at more than 6 weeks of $10 \mathrm{mg} / \mathrm{day}$ of atorvastatin, attending Endocrinology OPD in last 5 years, were included. Serum lipid profile at baseline and after statin treatment were estimated using commercial kits on Beckman Coulter DXC 600 auto analyzer. Serum LDL-C was calculated using Friedewald formula. Institutional research and ethics committees were approved this study.

Data was presented as mean $\pm \mathrm{SD}$. Comparison of continuous variables was done in the same cohort of patients at 2 points of time with 'paired $t$ test'. $\mathrm{P}$ value of 0.05 or less was considered as significant. The data was analysed statistically using the IBM Statistical Package for the Social Sciences (SPSS Inc., Chicago, IL, USA, version 20)

\section{Results:}

Total 373 cases were reviewed. Thirty-five cases, who did not have complete data, had some secondary cause of dyslipidemia and who were taking other lipid lowering agents along with atorvastatin were excluded. Finally, 338 cases were analyzed. Patients were $60.5 \pm 9.0$-year-old with male to female ratio of 1.17. The mean body mass index among men and women were $26 \pm 3.2$ and $27.3 \pm 4.7 \mathrm{~kg} / \mathrm{m}^{2}$ respectively.

Pretreatment total cholesterol (TC), triglycerides (TG) and high-density lipoprotein cholesterol (HDL-C) and LDL-C was $195 \pm 35 \mathrm{mg} / \mathrm{dL}, 157 \pm 67 \mathrm{mg} / \mathrm{dL}$, $38.6 \pm 7.4 \mathrm{mg} / \mathrm{dL}$ and $126 \pm 30 \mathrm{mg} / \mathrm{dL}$ respectively. After treatment TC, TG and LDL-C were significantly (p value $<0.001$ ) reduced to $142 \pm 26 \mathrm{mg} / \mathrm{dL}, 135 \pm 57$ $\mathrm{mg} / \mathrm{dL}$ and $75 \pm 23 \mathrm{mg} / \mathrm{dL}$ respectively. However, there was no change in HDL-C. The LDL-C reduced by 40\% from baseline. Moderate (30-50\%) and high intensity reduction $(\geq 50 \%)$ of LDL-C observed in $51.2 \%$ and $23 \%$ respectively.
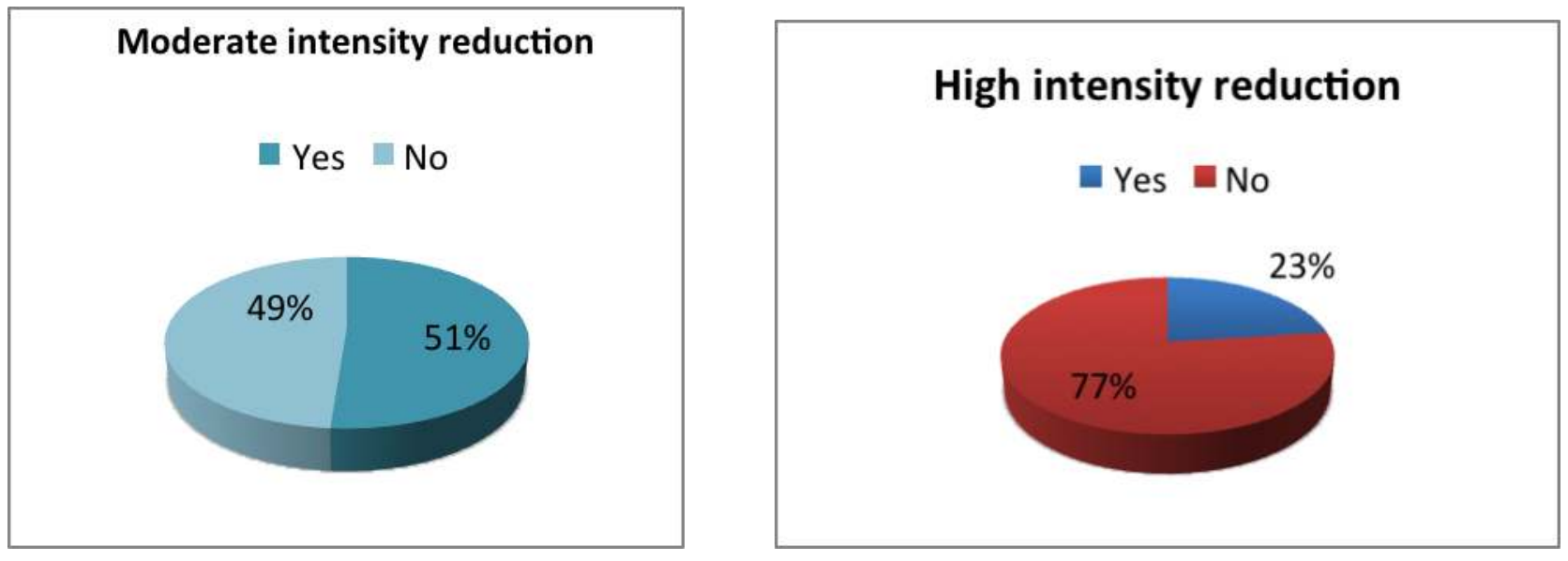

\section{Discussion:}

With low dose (10 mg/day) atorvastatin, moderate intensity reduction of LDL-C was observed in about half of the cases and high intensity reduction in about quarter of the cases. According to ACC/AHA 2013 guidelines, high intensity reduction of LDL-C requires 40-80mg of atorvastatin ${ }^{2}$. Even with minimal dose of atorvastatin, around a quarter of the patients reached high intensity of reduction of LDL-C. It showed that quite good number of cases reached defined reductions in LDL-C. Based on the response with low dose, further increase in the dose of statin can be done in required cases. This will help in reduction of side effects and cost burden to the patients. The present study showed that in patients with type 2 diabetes mellitus, $10 \mathrm{mg}$ of atorvastatin daily was effective in reducing LDL-C to defined intensity targets.

\section{Reference:}

1. Preis SR, Hwang SJ, Coady S, Pencina MJ, D' Agostino RB, Savage PJ, et al. Trends in all-cause and cardiovascular disease mortality among women and men with and without diabetes mellitus in the Framingham Heart Study, 1950 to 2005. Circulation 2009;119:1728-1735.

2. Stone NJ, Robinson J, Lichtenstein AH, BaireyMerz CN, Blum CB, Eckel RH, et al 2013 ACC/AHA guideline on the treatment of blood cholesterol to reduce atherosclerotic cardiovascular risk in adults: a report of the American College of Cardiology/American Heart Association Task Force on Practice Guidelines. Circulation. 2013;00:000-000. 